\title{
Robótica educativa desde la investigación como estrategia pedagógica apoyada en tic en la escuela
}

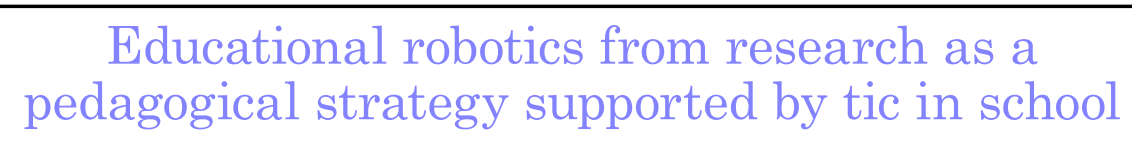

DOI: http://dx.doi.org/10.17981/cultedusoc.9.3.2018.82

Artículo de investigación. Fecha de recepción: 15/06/2018. Fecha de aceptación: 27/11/2018

\author{
Farides Fontalvo Caballero ${ }^{1}$ \\ Adargenis Santoya-Mendoza; Ailene Díaz-Mercado; Farides Fontalvo-Caballero; \\ Ledys Daza-Torres; Luis Avendaño-Bermúdez; Luz Sánchez-Noriega; \\ Pablo Ramos-Bernal; Elda Barrios-Martínez; María López-Daza; \\ Gloria Osorio-Cervantes; Martha Rodríguez-Pertuz; Velma Moreno-Polo² \\ Institución Educativa Departamental Gabriel García Márquez (Colombia) \\ falicape0416@hotmail.com
}

Para citar este artículo:

Santoya-Mendoza, A., Díaz-Mercado, A., Fontalvo-Caballero, F., Daza-Torres, L., Avendaño-Bermúdez, L., Sánchez-Noriega, L., Ramos-Bernal, P., Barrios-Martínez, E., López-Daza, M., Osorio-Cervantes, G., Rodríguez-Pertuz, M. y Moreno-Polo, V. (2018). Robótica educativa desde la investigación como estrategia pedagógica apoyada en tic en la escuela. Cultura. Educación y Sociedad 9(3), 699-708. DOI: http://dx.doi.org/10.17981/cultedusoc.9.3.2018.82

\section{Resumen}

La robótica educativa es un medio de aprendizaje basado en el diseño y construcción de objetos controlados a través de sistemas computacionales, convirtiéndose en un escenario donde los estudiantes estimulan la capacidad de creación. El propósito del estudio fue implementar la robótica educativa desde la investigación como estrategia pedagógica apoyada en TIC en la escuela. Este estuvo guiado bajo los lineamientos metodológicos de la IEP con un diseño basado en las trayectorias de indagación. La población estuvo conformada por cuarenta (40) estudiantes de la IED Gabriel García Márquez del Municipio de Aracataca, Como técnicas de recolección de datos se utilizaron el diario de campo y la observación. Se evidencio que los estudiantes apropian el conocimiento a partir de actividades prácticas donde la integración la investigación permite realizar el proceso de asociación entre la teoría y la aplicación de la misma en el contexto social donde se desenvuelve el estudiante.

Palabras clave: Robótica educativa, investigación como estrategia pedagógica, tecnologías de la información y la comunicación.

\section{Abstract}

Educational robotics is a learning medium based on the design and construction of controlled objects through computer systems, becoming a scenario where students stimulate the ability to create. The purpose of the study was to implement educational robotics from research as a pedagogical strategy supported by ICT in the school. This was guided under the methodological guidelines of the IEP with a design based on the trajectories of inquiry. The population consisted of forty (40) IED students Gabriel Garcia Marquez from the Municipality of Aracataca, chosen through stratified random sampling taking as criteria of inclusion the grade level and the working day. Data logging and observation were used as data collection techniques. It was evidenced that the students appropriate the knowledge from practical activities where the integration of the research allows to realize the process of association between the theory and the application of the same in the social context where the student develops, generating in this way a significant learning.

Keywords: Educational robotics, research as a pedagogical strategy, information and communication technologies.

1 Líder del grupo "TIC y Robótica".

2 Docentes de la Institución Educativa Departamental Gabriel García Márquez, Grupo institucional, pertenecientes al grupo de investigación "TIC y Robótica".

- The author; licensee Universidad de la Costa - CUC. Cultura, Educación y Sociedad vol. 9 no. 3, pp. 699-708. Diciembre, 2018 Barranquilla. ISSN 2389-7724 Online 


\section{Introducción}

La educación ha presentado cambios significativos en la actualidad, implementando modelos pedagógicos que permiten el desarrollo integral del estudiante, introducir la investigación al aula de clase desde la educación básica, dota a los estudiantes de bases sólidas que articulen la comprensión y adaptación con la educación formal para el desarrollo de trabajos de grado, por lo cual el estudio busco implementar la Robótica Educativa la robótica educativa desde la investigación como estrategia pedagógica apoyada en TIC en la escuela, para el fomento de una educación en ciencia tecnología e innovación.

La robótica educativa ha crecido muy rápidamente en la última década en la mayoría de los países, su importancia radica en que cada día en nuestra vida cotidiana se están incorporando en ese sentido industrias, sistema de educación en todos los ámbitos, con el único propósito en utilizar esta herramienta pertinente a la robótica como medio de enseñanza y como medio de simplificar algunas actividades a diferentes niveles de enseñanza, que va más allá de adquirir conocimiento en el campo, en cierto modo lo que pretende es trabajar en el alumno competencias básicas que son necesarias en la sociedad de hoy día, como son; el aprendizaje colaborativo, la toma de decisión en equipo, entre otras funciones que hacen de la robótica una herramienta de gran valor en todos los sentidos (Secretaria de educación pública y cultura, 2014).

Así mismo, a robótica educativa se concibe como un contexto de aprendizaje que se apoya en las tecnologías digitales, en el diseño y construcción de creaciones propias, construidas con diferentes materiales y controladas por un computador llamadas simulaciones o prototipos. Estas creaciones pueden tener su origen, en un referente real, como; un proceso industrial automatizado, en el que los estudiantes recrean desde la apariencia su imaginación mediante máquinas para de interactuar con el ambiente, en un contexto para llegar a la simulación, o prototipos que corresponden a diseños totalmente originales, como; el diseño y control de un producto que resuelve un problema particular de la escuela, hogar, comunidad o de una industria (Acuña, 2003).

Las dinámicas sociales, avances tecnológicos, educativos y necesidades de la población tienden a cambiar mucho más rápido que los planes académicos que se imparten en las aulas. A medida que surgen nuevas tecnologías, se requiere de nuevas competencias que deben ser aprendidas por los estudiantes para integrarse a dichos cambios. Se ha demostrado que la introducción temprana de tecnología para la enseñanza de distintas áreas en este caso la robótica permite que en grados posteriores se puedan enseñar fases más avanzadas de los mismos conceptos llegando a comprensiones más profundas, y que permiten el que el alumno tener la facilidad en desarrollar habilidades con un sentido de creatividad necesario para el desarrollo económico del pais (Moreno, 2012)

Por su parte, la inclusión de las TIC en la educación ha llevado a los docentes a replantearse sus roles, estos influyen directamente en el proceso enseñanzaaprendizaje, y dichos cambios o avances que se han presentado a través del tiempo en el proceso educativo han permitido que se utilicen las herramientas tecnológicas como material de apoyo para el desarrollo de estrategias innovadoras con la finalidad generar motivación en los estudiantes, factor importante y necesario para fomentar un aprendizaje significativo (Acuña, 2003).

En Colombia, se ejecutó un proyecto titulado; la robótica educativa como estrategia didáctica sostenible, la cual busco incursionar los sistemas tecnológicos mediante la robótica educativa, ofreciendo el diseño de una estrategia de aprendizaje 
novedoso en el que se pudieran recrear a niños y adolescentes de los estratos 1 y 2 de la ciudad de Bucaramanga (Mora, y Prada, 2016).

En Bogotá, se presentó una investigación titulada; Robótica Educativa para estudiantes de ciclo dos, la presente abordó el tema de la enseñanza de la robótica educativa en las aulas del país, reconocida como una herramienta propia de las tecnologías de la formación y la comunicación TIC, incorporándose en el aula como instrumento que facilita los procesos de enseñanzaaprendizaje, ha tenido un gran auge debido a que se incrementa considerablemente la atención del estudiante por la influencia multisensorial, además de ello la facilidad de acceso a la información proporcionando al estudiante otras dimensiones que antes no podían ser concebidas (Rojas, 2016).

Se sabe que la educación ha presentado cambios significativos a través del tiempo, donde el maestro ha ejercido nuevos roles, $\mathrm{y}$ surge la inclusión de nuevas herramientas tecnológicas, donde emergen las Tecnologías de la Información y las Comunicaciones (TIC) como una alternativa a la que pueden acceder los alumnos como fuente de información, dichas herramientas tecnológicas, brindan al cuerpo docente un sin número de estrategias educativas que permiten dinamizar el currículo.

El presente estudio buscó implementar la Robótica educativa desde la IEP apoyada en TIC en la IED Gabriel García Márquez de Aracataca, donde los estudiantes diseñen creaciones propias (objetos que poseen cuerpo, control y movimiento), construidas con diferentes materiales y controladas por un computador llamadas simulaciones o prototipos. Transversalizando dicha estrategia a las diferentes áreas del conocimiento como; matemáticas, español, biología, historia y tecnología, fomentando la participación activa de los estudiantes, en ambientes de aprendizaje, creación, ciencia, tecnología e innovación.

\section{Robótica educativa desde la IEP apoyada en TIC en la escuela}

Se considera la robótica educativa como un área de la tecnología con amplia aceptación en la población escolar, permitiendo la exploración, creación y aprendizaje de contenidos programáticos de manera práctica, es un medio que logra desarrollar niveles de comprensión y entendimiento, estimulando la capacidad de solucionar problemas adaptados a la realidad y al futuro de la sociedad (Rojas, 2016).

La robótica educativa es definida como un medio de aprendizaje basado en diseño construcción de objetos con cierta similitud a los seres humanos y animales. Estos objetos pueden llegar a ser controlados a través de sistemas computacionales. De esta manera la robótica educativa se convierte en un escenario donde los estudiantes desarrollan capacidades a través de estrategias pedagógicas, partiendo desde la concepción de objetos que pueden suplir una necesidad, creación del objeto, ensamble y puesta en funcionamiento. El estudiante debe llegar a encontrar cierta armonía entre el hardware y el software, ya que los movimientos que realizan los robots tienen una intencionalidad y son un acople entre lo físico y lo lógico (Rojas, 2016).

Ocaña (2015) define la robótica educativa como un conjunto de actividades pedagógicas que fortalecen áreas específicas del conocimiento y desarrollan competencias en los estudiantes, a través de la creación, ensamble y el funcionamiento de robots. El objetivo de enseñar robótica, es lograr la adaptación de niños y adolescentes a procesos productivos actuales.

Según Pozo (2005) La robótica educativa es propicia para apoyar habilidades productivas, creativas, digitales y comunicativas; convirtiéndose en un motor para la innovación cuando produce cambios en las personas, en las ideas y actitudes, en las relaciones, modos de actuar y pensar de los estudiantes y educadores. La robótica im- 
plica progreso y desarrollo tecnológico. La apropiación del conocimiento en este campo es clave, para conseguir altos niveles de competitividad y productividad a futuro en un país. Uno objetivo de la enseñanza en robótica, es crear en estudiantes interés por las ciencias y la ingeniería desde la educación básica, teniendo en cuenta que su diversificación crece constantemente (Comité Español de automática, 2011).

Se define entonces la robótica educativa como un espacio apoyado en tecnologías digitales que involucra procesos de mediación pedagógica para que los estudiantes construyan y programen prototipos a partir del ingenio y la creatividad. Es una oportunidad donde a través de la experimentación obtienen lo que desean, donde el docente posee el rol de formador (Rojas, 2016).

Según Ruiz (2007), la robótica educativa permite a los estudiantes desarrollar habilidades como; pensamiento reflexivo, obtención de información, trabajo en equipo y la anticipación; al igual que competencias innovadoras, científicas, investigativas, culturales, tecnológicas, comunicativas y de liderazgo. Todo esto a partir del diseño de robots educativos al servicio de una comunidad educativa y de la sociedad. Integrando los siguientes componentes:

Por otro lado, la investigación es un proceso sistemático, organizado y objetivo, cuyo propósito es responder a una pregunta o hipótesis, aportando al aumento del conocimiento sobre un tema desconocido o poco estudiado. Así mismo, la investigación es una actividad sistemática dirigida a obtener, mediante observación y experimentación, nuevo conocimiento necesario para ampliar información en diversos campos de la ciencia y la tecnología (Hernández, Fernández y Baptista, 2006) por otro lado, la investigación se puede definir también como la acción y el efecto de realizar actividades intelectuales y experimentales de modo sistemático con el propósito de aumentar los conocimientos sobre una determinada materia y teniendo como fin ampliar el conocimiento científico (Roblero,2006)

De esta manera se comprende que la investigación posibilita la construcción de conocimiento, convirtiéndose en un instrumento para comprender una realidad o plantear soluciones frente a una problemática. Sin embargo, si integra a la educación se constituye como una base de la enseñanza, permitiendo al docente desde una reflexión crítica de la práctica pedagógica, elaborar nuevas estrategias que dinamizan el currículo, a su vez estas estrategias pueden dirigir procesos reales de transformación, no solo de prácticas pedagógicas sino también prácticas sociales inmersas en la escuela (Salcedo, 2000).

La investigación como estrategia pedagógica ejerce un impacto positivo al integrarla al aula de clases, brindándole a la comunidad educativa la posibilidad de construir nuevo conocimiento apropiando herramientas científicas, que respondan a una necesidad o problemática social, proyectando al estudiante hacia un aprendizaje significativo (Ausubel, 2002).

Así mismo, la Investigación como Estrategia Pedagógica, tiene como finalidad instaurar bases científicas a los estudiantes en el aula de clases, esta parte de una dinámica metodológica, que busca dar respuestas a los interrogantes de los niños y niñas de la Institución y que asigna a los docentes un nuevo rol en el aula, para que en el momento indicado, puedan ser ellos mismos quienes busquen comprender el mundo que los rodea e interpretar y describir sus problemas en un lenguaje sustentado en la ciencia. En la implementación de la IEP, se parte de la pregunta del sentido común de los niños, niñas y jóvenes para reelaborarla en la perspectiva de la educación popular, que reconoce la existencia de saberes comunes y elaborados así en la negociación con los conocimientos disciplinares, y de las maneras como se correlacionan en la negociación cultural (Mariño, 2010), 
La educación a lo largo del tiempo ha presentado cambios significativos, que invitan al docente a diseñar e implementar nuevas estrategias pedagógicas para dirigir el proceso de enseñanza con sus estudiantes, una estrategia pedagógica es un conjunto de actividades que se realizan con un orden lógico y coherente en función del cumplimiento de objetivos del plan académico. Es decir, es una planificación que contiene métodos o acciones que permiten al estudiante alcanzar los logros propuestos, mejorar su aprendizaje y facilitar su crecimiento personal (Picardo, Balmore, y Escobar, 2004).

Cochran y Litle (2006), plantearon la existencia de conocimientos y reflexiones en la acción, lo que permite integrar en las actuaciones explícitas e implícitas lo cognitivo, lo emocional, la teoría y la práctica. Reconociendo que tanto quien enseña como quien aprende dentro de una comunidad trabajan para generar conocimiento local, prever su práctica y teorizar sobre ella, interpretando las conclusiones de otros, todo lo anterior es posible al integrar la investigación al aula.

Por su parte los proyectos educativos se convierten en un instrumento de enseñanza con enfoque global, que toma en cuenta los componentes del currículo, sustentándose en las necesidades de la comunidad educativa. El proyecto educativo como herramienta para administrar el currículo, constituye una forma de organizar sistemáticamente el aprendizaje, involucrando directamente a los actores del proceso, aportando un carácter transversalizador a todas las áreas del conocimiento (Carrillo, 2001).

De esta manera es importante que el docente a partir del PEI, el currículo y el plan de aula dirija su proceso de enseñanza utilizando la robótica educativa desde la investigación como estrategia pedagógica apoyada en TIC, para lo cual la metodología del proyecto se construye, en una diná- mica en la que los grupos por edad y por intereses convierten sus preguntas de sentido común en problemas de investigación, donde a través del proceso de indagación, se aclaran conceptos utilizando herramientas tecnológicas, vinculados a los contenidos curriculares. Los proyectos educativos se apoyan en un enfoque holístico que resalta la necesidad de desarrollar metodologías que tengan en cuenta los contextos, la vida y los intereses de los participantes en la actividad escolar, funcionando como un puente entre la pedagogía, la investigación y la vida (Salcedo, 2000).

Las organizaciones, señalan Mendoza, López y Salas (2016), "son entes que generan sus propios objetivos y diseñan la forma como alcanzarlos teniendo en cuenta, necesariamente, las influencias del entorno social" (p. 62). Las escuelas no escapan de ello y es de allí que, en las organizaciones cualquiera que sea su ámbito, se hace preciso introducir una gestión estratégica como una preocupación por posicionarse. (Mendoza, López y Salas, 2016)

La investigación como estrategia, en las escuelas, debe ser concebida similar al pensamiento estratégico que según Arellano (2004) citado por Mendoza y López (2015), "hace referencia a la forma del pensamiento que se dirige hacia la creatividad, imaginación, y búsqueda de opciones innovadoras y realistas, como potenciación de lo posible enmarcado dentro de un proyecto de construcción intencional de la realidad". (p. 154)

Una de las finalidades de la educación es capacitar a los estudiantes para comprender, crear y participar en la construcción de su conocimiento, para lo anterior el docente debe cumplir un rol de guía o formador, que a través de estrategias pedagógicas motive al estudiante a alcanzar los logros propuestos, incluir en dichas estrategias las TIC, servirá como una herramienta tecnológica que muestra una forma diferente de organizar, representar y codificar la realidad, 
además estas son instrumentos que permiten un grado de aplicación de los conocimientos adquiridos, con lo cual "se podrá avanzar en el conocimiento sobre las condiciones de creación y de permanencia de este tipo de empresas en el mercado y de manera más amplia, del dinamismo del sistema industrial" (Maculan, Jiménez y Castellanos, 2015, p. 16). En efectos, las tecnologías del aprendizaje representan una de las fuerzas renovadoras en los sistemas de aprendizaje y constituyen un elemento clave para el desarrollo de la educación y la formación (Oleagordia, s.f).

La educación a lo largo del tiempo ha presentado cambios significativos, que invitan al docente a diseñar e implementar nuevas estrategias pedagógicas para dirigir el proceso de enseñanza con sus estudiantes, entendiendo estrategia como un dinamismo que potencializa el crecimiento socioeconómico, que genera desarrollo e inclusión (Pacheco, 2013), y estrategia pedagógica como un conjunto de actividades que se realizan con un orden lógico y coherente en función del cumplimiento de objetivos del plan académico. La tecnología se puede definir como el medio mediante el cual se transmite conocimiento científico a la solución de problemas concretos de una manera efectiva. Donde el concepto tecnología implica crear competencias que se expresan en recursos tecnológicos, aparatos, procedimientos y habilidades (Rías, 2004).

En la actualidad las nuevas tecnologías de la información y comunicación (TIC) han tomado un gran auge y su integración al contexto educativo ha generado grandes cambios en el proceso de enseñanza, estas giran en torno a tres medios básicos: la informática, la microelectrónica y las telecomunicaciones; de manera interactiva e interconectadas, lo que permite conseguir nuevas realidades comunicativas, impactando de manera positiva la escuela. (Belloch, 2012). Al respecto,
Betancur, Carmona, Contreras, Karam, Maestre, Romero y Uribe (2014) resaltan la pertinencia de integrar las TIC al proceso educativo por cuanto desde su perfil de herramientas o entornos virtuales de aprendizaje viabilizan la mediación didáctica - pedagógica.

Las TIC son tecnologías para almacenar, recuperar, procesar y comunicar la información. Existe una variedad de recursos electrónicos que se encuadran dentro del concepto de TIC como; televisión, teléfonos, videos, ordenadores, entre otros. Sin embargo, los medios más representativos de la sociedad actual son los ordenadores que nos permiten utilizar diferentes aplicaciones informáticas (presentaciones, aplicaciones multimedia, programas ofimáticos y redes de comunicación (Belloch, 2012).

No obstante, el uso de las TIC no implica la implementación de una estrategia de enseñanza/aprendizaje. En algunos casos se producen procesos educativos que integran las TIC siguiendo una metodología tradicional en la que se enfatiza el proceso de enseñanza, donde el estudiante recibe la información del docente. No obstante, para guiar el proceso educativo, se puede fomentar la interacción, el aprendizaje colaborativo y el aprendizaje por descubrimiento utilizando las TIC basados en los diferentes recursos y servicios que ofrece Internet (Belloch, 2012).

En ese sentido la investigación como estrategia pedagógica apoyada en las TIC es una propuesta educativa que apunta a mejorar la calidad de la educación, presentando una formación en ciencia, tecnología e innovación. Que apoyada en los contenidos de la Robótica Educativa, permiten adquirir los conocimientos y logros del plan académico de las instituciones educativas a través de una metodología didáctica y práctica, favoreciendo e impactando de manera positiva el proceso enseñanza aprendizaje. 
Regularmente las situaciones que son valoradas como innovaciones surgen a partir de la necesidad de resolver problemas particulares o suplir necesidades y demandas. De igual forma, en el ámbito educativo la innovación se concibe como un proceso que permite incorporar cambios en las formas de hacer, pensar y vivir. "Una innovación es un proceso de gestión de cambios específicos, en ideas o instrumentos, hasta su consolidación (Espasa, 2005).

Para Ramírez (2018), la gerencia estratégica del talento humano, está facilitando nuevos estilos administrativos y operativos de labor organizacional para una generación de valor agregado, con un componente referenciado por un enfoque sistémico humanizante hacia lo estratégico - dinamizador, inclusión de la ciencia y tecnología, capacidad de internacionalización y asociatividad; convirtiendose en factores claves de competitividad, posibilitando permanencia en el contexto organizacional en un entorno globalizado.

\section{Metodología}

\section{Diseño}

La metodología del proyecto de investigación se realizó a partir de los recorridos de las trayectorias de indagación, basados en la integración de la IEP apoyada en TIC al aula, articulado al plan de estudios, desde las diferentes áreas se estructuraron temáticas relacionadas con la robótica educativa, la finalidad de este proceso investigativo, fue generar una trasformación social y educativa a través del aprendizaje en contexto, involucrando intereses, motivaciones y realidades de los estudiantes para generar conocimiento científico, donde se establecieron cinco (5) momentos que especifican en la ejecución y las metas trazadas para darle solución a la pregunta problema planteada.

\section{Participantes}

Participantes La unidad de análisis estuvo conformada por cuarenta (40) estudiantes pertenecientes a los grados sexto, séptimo y octavo de la Institución Educativa Departamental Gabriel García Márquez, con edades que oscilan entre los once (11) y quince (15) años, cabe destacar que los participantes realizaron el diligenciamiento del consentimiento informado para efectos de publicación de los resultados.

\section{Técnicas e instrumentos}

En cuanto a los instrumentos de recolección se utilizó el diario de campo, este es un instrumento que ayuda al investigador a inmiscuirse de forma total en el ambiente con el cual se va a encontrar, es un proceso de hechos, recolección de datos y análisis. El diario de campo es parte de un registro cronológico que se desarrolla mediante la sesión compartida con los infantes, es donde se describe detalladamente los aspectos positivos como negativos colocando significado a cada uno de ellos como y utilizados y utilizados. Un cuaderno donde se va a descubrir la manera de expresar y escribir frente a la relación que se tiene con los infantes, es un momento de no cohibir lo vivido y las experiencias obtenidas.

A su vez se empleó un cuestionario de 7 preguntas con dos opciones de respuesta, aplicada a los estudiantes participantes en la investigación con la finalidad de conocer sus actitudes frente a la implementación de nuevas estrategias en el contexto educativo, Para el diseño del instrumento se realizó una reunión del grupo de investigación donde se escogieron las preguntas más adecuadas para el mismo.

\section{Procedimiento}

A continuación, se presentan los diferentes momentos. 
- Momento (1): selección y análisis de la población sujeto de estudio.

- Momento (2): integración de la temática mediante la IEP al aula de clases, se integraron grupos estudiantiles para seleccionar problemáticas relacionadas con la Robótica educativa. Los estudiantes se plantearon preguntas y con el acompañamiento del docente realizaron el proceso de indagación mediante herramientas virtuales para luego realizar el proceso de construcción de sus prototipos.

- Momento (3): socialización de los resultados de las indagaciones de estudiantes en una feria institucional dirigida por los docentes a cargo.

- Momento (4): análisis de los resultados de la implementación de la estrategia a través de la observación y diario de campo.

- Momento (5): propagación y divulgación de los resultados.

\section{Resultados}

Para la implementación de la Robótica educativa mediante la IEP apoyada en TIC al aula se dirigieron clases desde el área de informática donde los estudiantes tenían la libertad de escoger inventos creados por el hombre, a partir de esta premisa los jóvenes realizaron un proceso de investigación sobre el objeto, plantearon su problema de investigación, seguidamente, definieron sus instrumentos de recolección de información, donde realizaron el recorrido de las trayectorias de indagación, el cual les sirvió para la construcción de su prototipo o simulación, además, estos debían ser elaborados con materiales reciclables, donde los recursos otorgados por la institución serian herramientas para la construcción de los mismos, finalmente los resultados de sus proyectos se fueron expuestos entre los integrantes del grupo y unos jueces expertos, como estrategia para la apropiación social del conocimiento.
En cuanto a la triangulación de los resultados arrojados por el cuestionario y la observación registrada en el diario de campo, se observa que los estudiantes presentan actitudes positivas frente a la implementación de nuevas estrategias en el contexto educativo, puesto que mediante el ejercicio realizado por los docentes frente la implementación de la Robótica educativa mediante la IEP apoyada en TIC lograron descubrir nuevas habilidades, los participantes manifestaron que es más fácil aprender la teoría si se lleva a la práctica en forma didáctica. Afirmaron que les gusta la investigación, y se sienten motivados si los docentes utilizan nuevas estrategias metodológicas. Por su parte los participantes comprenden mejor las clases si buscan información e indagan en diferentes fuentes, no les gusta que los docentes dirijan las clases de manera magistral y prefieren realizar proyectos de investigación donde indaguen sobre una temática relacionada de la clase, empleando la robótica o las TIC (tabla 1).

\section{TABLA 1}

Respuestas del cuestionario

\begin{tabular}{lcc}
\hline Pregunta & Si & No \\
\hline $\begin{array}{l}\text { ¿Desde tu experiencia en el proyecto, } \\
\text { piensas que con la robótica educativa } \\
\text { puedes aprender los conocimientos de } \\
\text { diferentes asignaturas? }\end{array}$ & 35 & 5 \\
$\begin{array}{l}\text { ¿Crees que es más fácil aprender la } \\
\text { teoría, si la pones en práctica? }\end{array}$ & 40 & 0 \\
$\begin{array}{l}\text { ¿Te gusta la investigación? } \\
\begin{array}{l}\text { ¿Te sientes motivado cuando un } \\
\text { docente utiliza nuevas metodologías } \\
\text { en clase? }\end{array}\end{array}$ & 40 & 0 \\
$\begin{array}{l}\text { ¿Buscar información e indagar } \\
\text { en diferentes fuentes, te ayuda a } \\
\text { comprender mejor las clases? }\end{array}$ & & \\
$\begin{array}{l}\text { ¿Te gusta que los docentes dirijan las } \\
\text { clases de forma magistral? }\end{array}$ & 60 & 10 \\
$\begin{array}{l}\text { ¿Prefieres realizar proyectos de } \\
\text { investigación donde indagues sobre } \\
\text { una temática relacionada de la clase, } \\
\text { empleando la robótica o las TIC? }\end{array}$ & 40 & 0 \\
\hline
\end{tabular}

Fuente: elaboración propia. 


\section{Conclusiones}

La robótica educativa como un espacio apoyado en tecnologías digitales (TIC) que involucra procesos de mediación pedagógica para que los estudiantes construyan y programen prototipos a partir del ingenio y la creatividad. Es una oportunidad donde a través de la experimentación obtienen lo que desean, donde el docente posee el rol de formador. La robótica educativa implementada en las instituciones educativas departamentales permite a los estudiantes desarrollar habilidades como; el pensamiento reflexivo, la obtención de información, el trabajo en equipo y la anticipación; al igual que las competencias innovadoras, científicas, investigativas, culturales, tecnológicas, comunicativas y de liderazgo.

El desarrollo de actividades dentro del aula empleando la robótica como una estrategia pedagógica facilita en los estudiantes el desarrollo de sus competencias para seleccionar, adaptar tecnologías, indagar e implementar a esta dentro de la consecución de la satisfacción de las necesidades humanas, a partir de costumbres que ayudan a una cultura ambiental con propósitos éticos, que apoyado en la IEP se convierte en un espacio para la reflexión, la construcción de conocimiento y la divulgación o socialización del mismo, por medio de ferias científicas. desarrolladas en la institución, en el municipio o incluso en el departamento, donde se puedan visionar las creaciones innovadoras de nuestros estudiantes.

\section{Referencias}

Acuña, A. (2003) La robótica educativa: un motor para la innovación. Recuperado de http://www.fod.ac.cr/robotica/ descargas/roboteca/articulos/2007/roboticamotor_innova_articulo.pdf

Ausubel, D. (2002) Adquisición y retención del conocimiento. Madrid: Ed. Paidós.
Belloch (2012). Las Tecnologías de la Información y Comunicación en el aprendizaje. Recuperado de https://www.uv.es/ bellochc/pedagogia/EVA1.pdf

Betancur, S., Carmona, L., Contreras, R., Karam, J., Maestre, N., Romero, Y., \& Uribe, S. (2014). Videojuegos y tic como Estrategias Pedagógicas: Formación para el uso seguro de internet. Cultura Educación y Sociedad, 5(1). Recuperado a partir de https://revistascientificas. cuc.edu.co/culturaeducacionysociedad/ article/view/997

Carrillo, T. (2001) El proyecto pedagógico de aula. Educere, 5(5). 335-344.

Cochran, M. y Litle, S. (2003). Más allá de la certidumbre adoptar una actitud indagadora sobre la práctica. Barcelona: Octaedro.

Comité Español de automática-CEA. (2011). El libro blanco de la robótica en España: Investigación, tecnologías y formación. España: CEA-GTRob-MEC. Recuperado de http://www.ceautomatica.es/wp-content/uploads/2015/08/LIBRO-BLANCO-DE-LA-ROBOTICA-2_v2.pdf

Gallego, E. (2005). Técnicas para la Implementación de la Robótica en la Educación Primaria. Recuperado de http:// complubot.educa.madrid.org/actividades/inrerdidac_robotica_primaria.pdf.

Hernández, R., Fernández, C. y Baptista, P. (2010). Metodología de la investigación. México, D.F.: Editorial Mc Graw Hill.

Jiménez, M. y Cerdas, R. (2014). La robótica educativa como agente promotor del estudio por la ciencia y la tecnología en la región atlántica de Costa Rica. Congreso Iberoamericano de Ciencia, Tecnología, Innovación y Educación. Buenos Aires, Argentina.

Maculan, A., Jiménez, C. y Castellanos, O. (2015). Aprendizaje en el proceso de incubación de empresas de base tecnológica. Económicas CUC, 36(1), 15-48. 
Mariño, G. (2010). El diálogo en la educación de jóvenes y adultos. Dos propuestas pedagógicas para implementarlo. En: $E l$ taller dialógico / la recuperación de experiencias laborales. Bogotá, D.C.: OEI.

Mendoza, D. y López, D. D. (2015). Pensamiento estratégico: centro neurálgico de la planificación estratégica que transforma la visión en acción. Económicas $C U C$, 36(1), 153-179.

Mendoza, D., López, D. y Salas, E. (2016). Planificación estratégica de recursos humanos: efectiva forma de identificar necesidades de personal. Económicas CUC, 37(1), 61-79.

OEA. (2004). Las nuevas tecnologías y la educación inclusiva a la capacitación y actualización docente en la búsqueda de una educación de calidad. Buenos Aires: Ministerio de Educación Ciencia y Tecnología de la Nación de Argentina.

Oleagordia, I. (s.f.). Estrategias educativas para el uso de las nuevas tecnologías de la información y la comunicación. OEI Revista Iberoamericana de la educación, 9. 1-3

Pacheco, C. (2013). Estrategias empresariales más utilizadas por las pymes en Sincelejo. Económicas CUC, 34(1), 183-202.

Picardo, O., Balmore, R. y Escobar, J. (2004). Diccionario enciclopédico de ciencias de la educación. San Salvador: El Salvador.
Ramírez, R. (2018). Tendencias emergentes de la gestión de talento humano en las organizaciones. En, M. Quintero y M. Sánchez, Responsabilidad Corporativa: una mirada integral en América Latina. (101-107). Cali: Editorial Universidad del Valle.

Rojas, Y. (2016). Robótica educativa para estudiantes de ciclo dos. Barranquilla: Corporación Universitaria Minuto de Dios.

Salcedo, R. (2000) Experiencias docentes, calidad y cambio escolar: investigación e innovación en el aula la investigación en el aula: y la innovación. Recuperado de http://www.banrepcultural.org/blaavirtual/educacion/expedocen/expedocen8a. htm.

Salgado, A. (2007). Investigación cualitativa: diseños, evaluación del rigor metodológico y retos. Liber, 13(13). 71-78. Recuperado de http://www.scielo.org.pe/ pdf/liber/v13n13/a09v13n13.pdf

Secretaria de educación pública y cultura. (2014). Guía didáctica para el responsable del programa de robótica educativa. Departamento de tecnología educativa. Sinaloa.

Rias, V. (2004). A body of knowledge for management of technology (MOT-BOK). Recuperado de http://www.technoscan. com/pdf/02.25\%20-\%20BOK\%20article. $\operatorname{pdf}$ 